\title{
Sperm morphology differences associated with pig fertility
}

2

3 Mandawala $\mathrm{AA}^{1}$, Skinner $\mathrm{BM}^{2}$, Walling $\mathrm{GA}^{3}$, Fowler $\mathrm{KE}^{1^{*}}$ and Harvey $\mathrm{SC}^{1^{*}}$

4

$5 \quad{ }^{1}$ School of Human and Life Sciences, Canterbury Christ Church University, Canterbury, UK

$6{ }^{2}$ University of Cambridge, Cambridge, UK

$7 \quad{ }^{3}$ JSR Genetics, Southburn, Driffield, East Yorkshire, UK

8

$9 \quad{ }^{*}$ Fowler KE and Harvey SC are joint last authors

10

11 Corresponding author:

12 Dr. Simon Harvey,

13 School of Human and Life Sciences,

14 Canterbury Christ Church University,

15 Canterbury,

16 CT1 1QU

17 UK

18 Email: simon.harvey@canterbury.ac.uk

19 Telephone: +44 1227782327

20

21 Short title:

22 Pig sperm morphology analysis

23

24

25

26 


\section{Abstract}

28 Artificial insemination is routinely used in commercial pig breeding, for which the use of high quality

29 semen samples is imperative. Currently, semen quality is determined manually by morphological

30 assessment. This method leads to high inter-operator variability due to its subjective nature. The

31 development of a semi-automated software-based approach to assess sperm morphology would

32 enable faster identification of morphological defects and permit identification of subtle differences

33 that may affect fertilisation success. Here we have used a novel method to comprehensively analyse

34 pig sperm nuclear morphology in greater detail than was previously possible. Semen samples from 50

35 fertile and 50 sub-fertile samples that had been previously manually categorised as fertile or sub-

36 fertile were analysed using this new method, with at least 200 fixed and DAPI (4',6-diamidino-2-

37 phenylindole) stained sperm heads imaged per sample. Differences in sperm nuclear morphology

38 were observed between fertile and sub-fertile samples; specifically, fertile samples were associated

39 with higher mean nuclear area, a consequence of a greater head width and a lower variability between

40 sperm heads. This novel, unbiased and fast analysis method demonstrates a significant difference in

41 sperm head morphology between fertile and sub-fertile animals, and has the potential to be further

42 developed and used as a tool for sperm morphology assessment in the pig breeding industry.

44 Keywords: Morphology, morphometrics, nucleus, pig, sperm 


\section{Introduction}

47 Male fertility is a consequence of both the number and quality of sperm (Cooper et al., 2009). In technology (ART) procedures, such as in vitro fertilisation (IVF) and intracytoplasmic sperm injection (ICSI). In agriculturally significant species (for example, pigs, cattle and sheep), where a key goal is to maximise the production of meat at a low cost, male fertility is also a challenge (Tardif et al., 1999). To this end, improving reproductive traits is of paramount importance. In such species, the critical aim is often to identify sub-fertile animals quickly and cheaply so they can be removed from breeding schemes; boars from a nucleus herd with fertility problems have the potential to reduce litter sizes throughout the breeding population (O'Connor et al., 2017).

Artificial insemination (Al), is the oldest (Roca et al., 2006) and most routinely used technique in commercial animal breeding, especially in livestock species (Dziuk and Henshaw, 1958; Polge et al., 1968; Johnson et al., 1981; Singleton, 2001; Gerrits et al., 2005; Roca et al., 2006; Feitsma, 2009). Over the past three decades, the use of Al has benefited the pig breeding industry, particularly in Europe where over $80 \%$ of sows are bred through Al (Roca et al., 2006). In North America the technique is also widely implemented, especially in large farming units (Gerrits et al., 2005). The principal objective of $\mathrm{Al}$ in the pig breeding industry is to permit the dissemination of genetics from high genetic merit boars to as many sows as possible. Without Al more boars would be needed and hence animals of lower genetic merit would be required in breeding programmes. Moreover, the technique enables the opportunity to introduce superior genetic traits into sows whilst reducing the incidence of disease transmission, an advantage that does not exist with natural mating (Maes et al., 2008). Al is achieved by depositing spermatozoa into the female genital tract using artificial devices and processes. The standardised method of insemination is the intra-cervical insemination technique, with the semen dose deposited in the posterior region of cervical canal (Roca et al., 2006). 
In humans, semen analysis is widely used to evaluate male fertility in infertile couples (Rowe et al., 1993) and may also be used for the determination of reproductive toxicity in therapeutic and environmental agents (Apostoli et al., 1998; U.S. Environmental Protection Agency, 1996). Various physical characteristics of semen are assessed and whilst parameters such as volume, appearance, $\mathrm{pH}$ and viscosity are considered important (Maree et al., 2010), several studies have shown that sperm morphology is critical when determining semen quality and hence quantifying male fertility (MacLeod and Gold, 1951; Hartman et al., 1964; Eliasson, 1971; Menkveld and Kruger, 1996; Coetzee et al., 1998; Auger et al., 2016). Generally, the cut-off values of what is considered 'normal' vary and are dependent on the fertility clinic. However, the following benchmarks were published in the World Health Organisation's $5^{\text {th }}$ edition of "normal semen analysis": morphology ( $₫ 4 \%$ normal forms), total motility ( $\geq 40 \%$ ), vitality ( $\geq 58 \%$ live), sperm concentration $(\geq 15,000,000$ per $\mathrm{mL}$ ) and volume ( $\geq 1.5 \mathrm{~mL})$ (Rowe et al., 1993; WHO, 2010). To date, a number of studies have been performed to analyse semen composition (Huggins et al., 1942; Owen, 2005) and to establish the relationship between sperm quality and fertility in men (Paz et al., 1977; Overstreet and Katz, 1987; Martin and Rademaker, 1988; Perreault et al., 2003; Jung and Schuppe, 2007; Agarwal et al., 2008). One such study by Guzick and colleagues used several comparative semen analyses of fertile and infertile men, to determine the most appropriate measurements that could be used in the determination of fertility potential in men (Guzick et al., 2001). Here, it was established that whilst threshold values for sperm motility, concentration and morphology could be used in the classification of males into fertile, indeterminate fertility, or sub-fertile categories, these measures cannot be used independently for the diagnosis of male infertility (Guzick et al., 2001). In livestock species, a key contribution to successful fertilisation following Al is also the use of high quality semen during insemination. As such, routine assessment of semen quality is a standard process in the animal breeding industry (López Rodríguez et al., 2013). A issue is however that the mammalian ejaculate does not contain a homogenous population of spermatozoa (Holt and Van Look, 2004); in 
general analysis of boar semen, semen quality is considered "normal" if the frequency of abnormal sperm heads does not exceed $10 \%$. "Normality" can also be assumed if the frequency of abnormalities in acrosomes, mid-pieces, tails or proximal cytoplasmic droplets is less than either $5 \%$ each or $15 \%$ when combined (Saravia et al., 2007). Whilst some abnormalities such as the existence of distal cytoplasmic droplets are considered irrelevant to fertility assessment, other abnormalities are regarded as serious defects due to their ability to hinder fertilisation and cause infertility (Saravia et al., 2007). Some of these include decapitated spermatozoa, acrosomal plicae (knobbed defect), nuclear vacuoles (diadem defect), short tails (tail stump), coiled tails (dag defect) and corkscrewed mid-pieces (Saravia et al., 2007).

As well as studying the morphology of whole spermatozoa, a limited number of studies have focused on analysis of sperm nuclear morphometry in agricultural animals (Vicente-Fiel et al., 2013a, b). A number of measurements of sperm nuclei have been compared in cattle (Bos taurus taurus), sheep (Ovis orientalis aries), goats (Capra aegagrus hircus) and pigs (Sus scrofa domesticus) using computerassisted sperm morphometry analysis-F (CASMA-F) (Vicente-Fiel et al., 2013a, b). Whilst mean numerical values for area, perimeter, length and width were identified for each of the four species studied, the key findings of this study were that drying and fixation only has a minimal effect on sperm nuclear morphometry and that variations between morphometric parameters do exist between the sperm nuclei of each species (Vicente-Fiel et al., 2013a). A second study (Vicente-Fiel et al., 2013b) used a combination of the CASMA-F method and multivariate cluster analysis to identify subpopulations of spermatozoa within the same four species. Based on these nuclear morphometrics, three subpopulations, namely, large, small-elongated and small-round were identified. Whilst it had previously been shown that sperm shape differed between high and low fertility bulls (Ostermeier et al., 2001), such observations have not been made in pigs. Currently, manual morphological assessment of a semen sample requires the observation of at least 500 sperm heads per sample (Saravia et al., 2007) which is laborious and may lead to high inter-operator variability due to the 
subjective nature of this assessment method. Here, we demonstrate the use of a semi-automated software based approach to assess sperm head morphometrics in both fertile and sub-fertile pigs.

\section{Materials and methods}

\section{Semen collection}

129 Fresh ejaculated sperm samples from boars of various breeds including Large White, Landrace, White

130 Duroc, Hampshire and Pietrain were collected using the 'gloved hand method' (King and Macpherson, 131 1973), by trained staff at JSR Genetics Ltd.. Samples were stored in Duragen extender, supplemented with no less than: $500 \mathrm{IU}$ per $\mathrm{ml}$ streptomycin; $500 \mathrm{IU}$ per $\mathrm{ml}$ penicillin; $150 \mathrm{mg}$ per ml lincomycin; and $300 \mathrm{mg}$ per $\mathrm{ml}$ spectinomycin, diluted to 2.3 billion sperm per dose. Samples were stored at $17^{\circ} \mathrm{C}$ and were prepared within two days following collection.

\section{Sample preparation}

137 Prior to preparation of samples for this study, semen samples were identified as either fertile or sub-

138 fertile using a combination of computer assisted sperm analysis (CASA), followed by manual 139 assessment. Specifically, samples that had a normal morphology score of above $70 \%$ (obtained from 140 CASA) and a motility score of above 4 (motility was graded from 1 to 5, 1 being dead and 5 being 141 excellent) (subjective manual assessment) were graded as fertile and those falling below these criteria 142 were graded as sub-fertile.

14450 fertile and 50 sub-fertile samples were used in this study. $2 \mathrm{~mL}$ of each semen sample was 145 centrifuged at $300 \mathrm{~g}$ for 5 minutes at $17^{\circ} \mathrm{C}$. The supernatant was discarded, the pellet was resuspended in $1.5 \mathrm{~mL}$ of fixative solution $(100 \%$ methanol and $100 \%$ acetic acid, added dropwise at a

$1473: 1$ ratio) and centrifuged at $300 \mathrm{~g}$ for 5 minutes at $17^{\circ} \mathrm{C}$. The supernatant was discarded and the pellet 148 was re-suspended in $1.5 \mathrm{~mL}$ of fixative solution. $10 \mu \mathrm{L}$ of each sample was then dropped onto the centre 149 of the surface of a labelled (sample ID, date), steam-warmed slide, immediately followed by $10 \mu \mathrm{L}$ of 
fixative solution. Subsequently, slides were air-dried for 2 minutes before 1 drop of fluorescent DAPI (4',6-diamidino-2-phenylindole) was added to the centre of the slide. Prepared slides were air-dried in the dark, for at least 20 minutes prior to microscopy.

153

Image acquisition

An Olympus IX83 inverted fluorescence microscope equipped with CellSens Dimension version 1.9 of 200 nuclei were imaged (at 1000x magnification) per sample.

\section{Data analysis}

160 Images were analysed using the ImageJ plugin 'Nuclear Morphology Analysis' (see Skinner et al., 2018,

161 freely available under the GNU General Public License version 3 from

162 https://bitbucket.org/bmskinner/nuclear morphology/wiki/Home). The analysis software enables automated recognition of round or asymmetric nuclei within an image of interest, and subsequent morphological analysis of these nuclei. Initially developed for the analysis of mouse sperm (Skinner et al., 2018), we adapted the feature recognition to analyse pig sperm. The software generates a range of measures; in this study, sperm heads were measured for: Area; Perimeter; Bounding Height and

167 Bounding Width, the dimensions of the rectangle perfectly enclosing the nucleus when vertically oriented; Circularity, a measure between 0 and 1 indicating how circular the heads are, with 1 indicating a perfect circle; Minimum Width across the centre of mass; Aspect, the ratio produced from

170 height divided by width; and Variability, a per-nucleus measure calculated as the square root of the

171 sum-of-squares difference at each index between the nuclear profile and the dataset median profile,

172 normalised to the length of the median profile. Analysis was carried out using software version 1.13.5.

173 Initial correlation analysis was used to identify redundant variables. Non-redundant variables were

174 then further analysed. For initial comparisons between fertile and sub-fertile animals, means of each 
175 variable were compared by ANOVA in R version 3.4.4 (R Core Team, 2018), with the normality of

176 residual distribution assessed using scatter and quantile-quantile plots.

177

178 For the cluster analysis, samples were randomly allocated into two groups; a training group of 30

179 fertile and 30 sub-fertile animals and a test group of 20 fertile and 20 sub-fertile animals. Sperm heads

180 from the training group were used to determine if there were sperm of detectably different types.

181 This was done by cluster analysis, with Ward linkage, squared Euclidean distance and standardised

182 variables in Mintab v17. The representation of sperm heads from fertile and sub-fertile animals within

183 in each identified cluster was then compared to that expected by chance. The training group was then

184 used to investigate if identified clusters could be used to predict fertility status. This was done by using

185 the mean cluster value from the training set to define the starting partitions for a K-means clustering.

\section{Results}

188 Pig sperm are (mostly) symmetrical about the anterior-posterior axis. Without a distinctive hook, as 189 in mouse sperm, to act as a reference point, the tail attachment point was chosen to anchor the angle 190 profiles generated and orient nuclei. The tail attachment point is characterised by a 'dimple' in the

191 nucleus (Figure 1A, point 1). For consistent alignment of the nuclei, we placed the tail attachment 192 region directly below the centre of mass of the nucleus. The software output prior to statistical 193 analyses is summarised in Figure 1. This indicates how we generate an angle profile for a given sample 194 and how a consensus sperm head shape for the population is produced. These refolded sperm heads 195 are then used both to trace any identified abnormalities on a particular segment of the refolded nucleus back to the angle profile, and to calculate a range of sperm head parameters for analysis. 
A

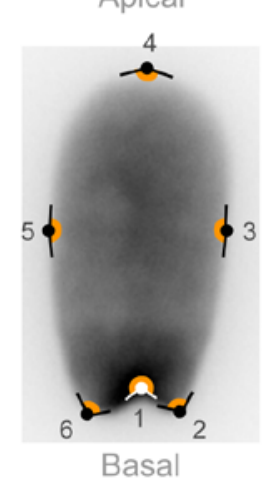

198
B

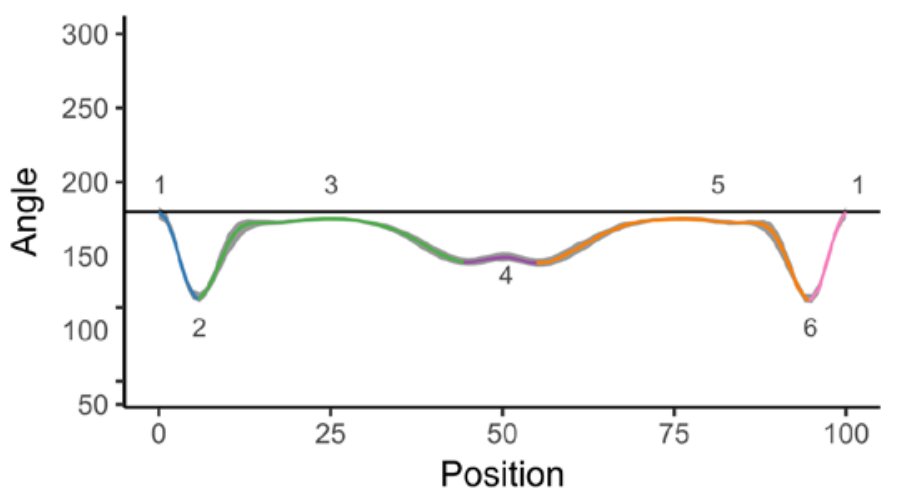

C

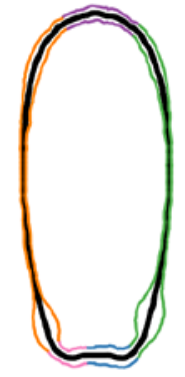

Figure 1: A representative summary of analysis workflow. (A) DAPI stained nucleus from a fertile boar sample, captured using an Olympus IX83 fluorescence microscope with pre-installed CellSens software at $1000 x$ magnification. The software measures the interior angles along the perimeter of the nucleus as represented by point 1-6. (B) Schematic showing how these angles generate a profile. The figure shows the median and interquartile rages for one fertile sperm sample, and has been segmented at local maxima and minima. (C) The consensus fertile pig sperm head shape, showing positions of profile segments in the nucleus.

Analysis of nuclear morphology from 50 fertile and 50 sub-fertile animals yielded measures from 11,534 and 11,326 nuclei, respectively. Correlation analysis of measured sperm head characters indicated that many of the measures were highly correlated (Table S1). Therefore, further analysis was undertaken using measures of Area, Circularity, Variability, Bounding Height and Bounding Width. Given the orientation of the sperm heads, Bounding Height and Width are subsequently referred to as Maximum Height and Width.

Comparisons between fertile and sub-fertile animals indicate that sperm heads differ according to fertility status, but that there is a large amount of variation between individuals (Figure 2A and B, and Figures S1-4). Analysis of mean trait values from each animal indicates that sperm heads from fertile animals have a higher overall area (Area, $\mathrm{F}_{1,98}=34.55, p<0.001$ ), are wider (Width, $\mathrm{F}_{1,98}=11.58, p=$ 
A

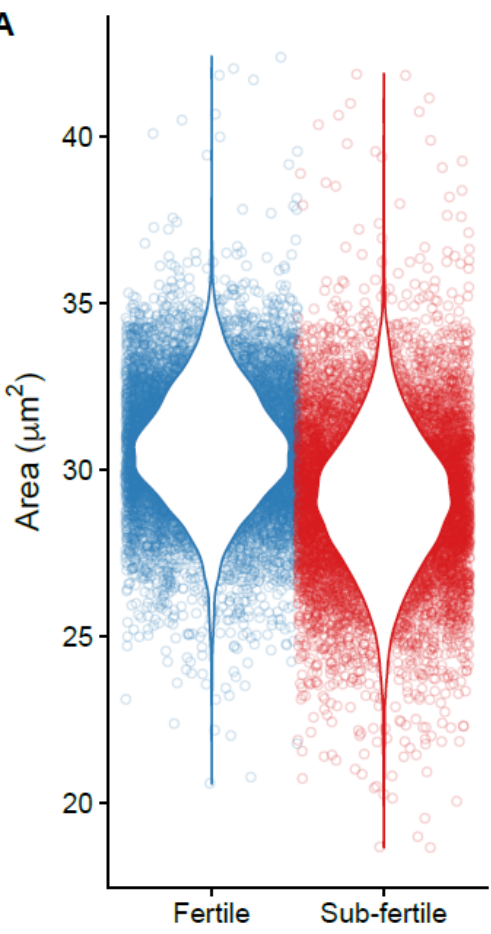

B

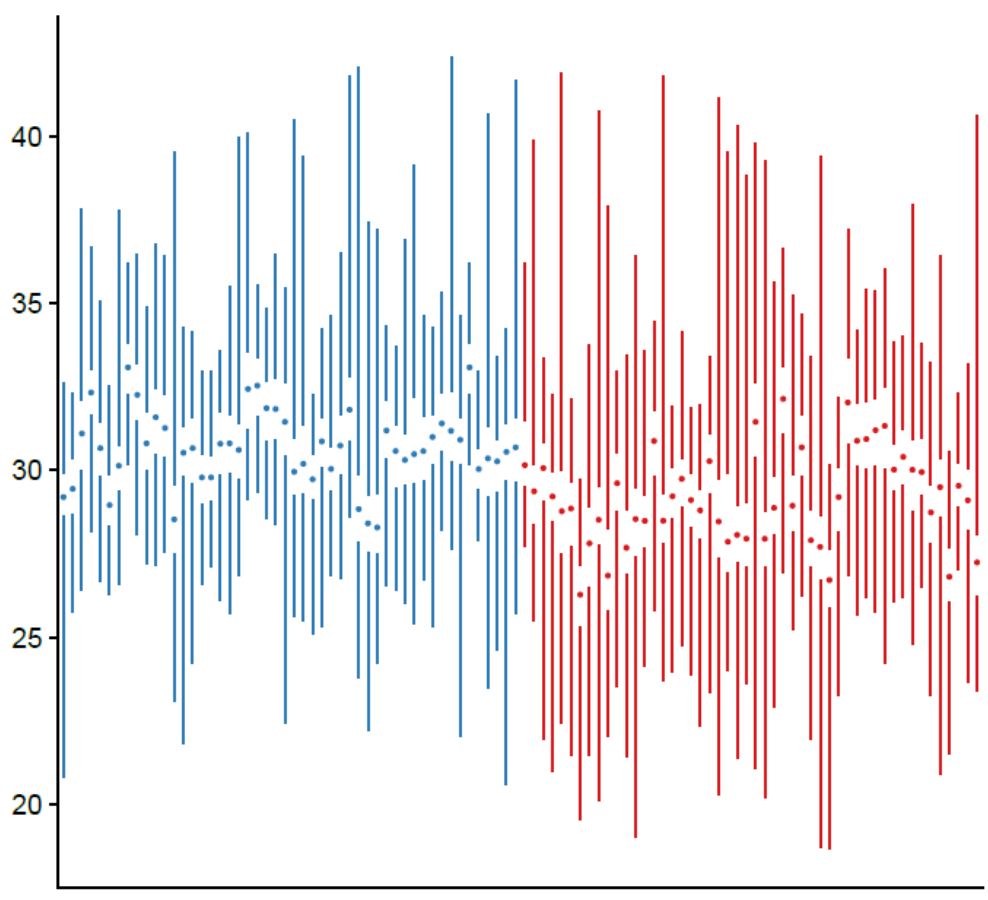

Figure 2: Comparison of sperm head area between 50 fertile (blue) and 50 sub-fertile (red) boars. (A)

Area of 11,534 sperm heads from fertile animals (blue) and 11,326 sperm heads from sub-fertile animals (red). (B) Individual Tufte boxplots of sperm head area for 50 fertile (blue) and 50 sub-fertile (red) boars.

We hypothesised that individual sperm samples would contain different nuclei types and that this may allow the identification of sub-fertile animals. For example, a certain sperm head type might only be found in sub-fertile animals or might be overrepresented within such animals. Such differences might represent abnormalities within these sub-fertile animals, which could also be used to investigate why their fertility was impaired, i.e. this could be informative about the biology. Additionally, the presence of differences of this type might allow the fertility of an animal to be predicted, i.e. this could allow the automated assessment of fertility. We therefore, randomly allocated samples into two groups: a 
training group of 30 fertile and 30 sub-fertile animals in which we investigated the presence, or not, of different nuclei types; and a test group of 20 fertile and 20 sub-fertile animals in which we investigate the ability of this type of analysis to predict fertility status.

In the training group, cluster analysis (Ward linkage, squared Euclidean distance and standardised

A

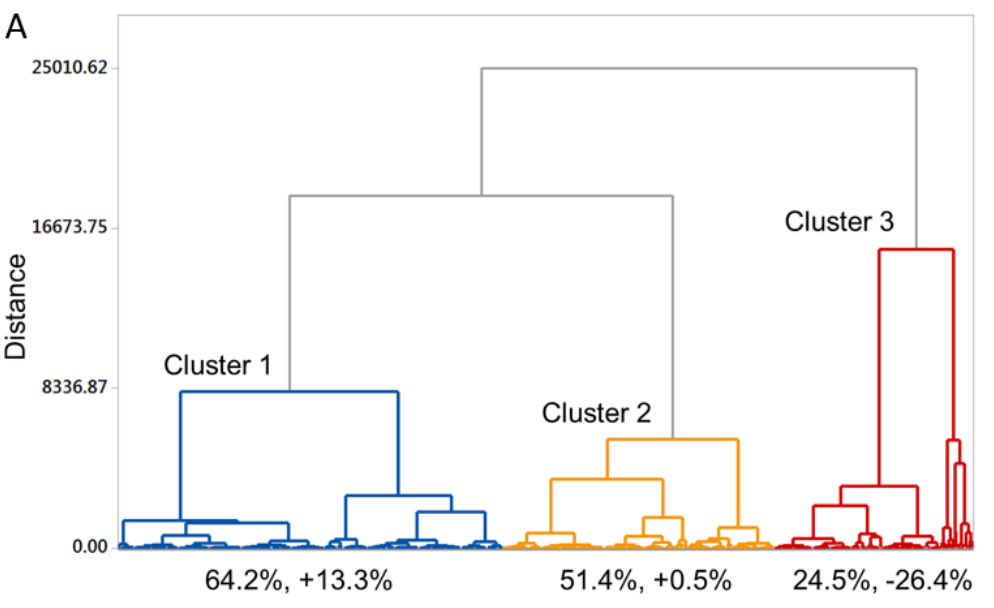

B

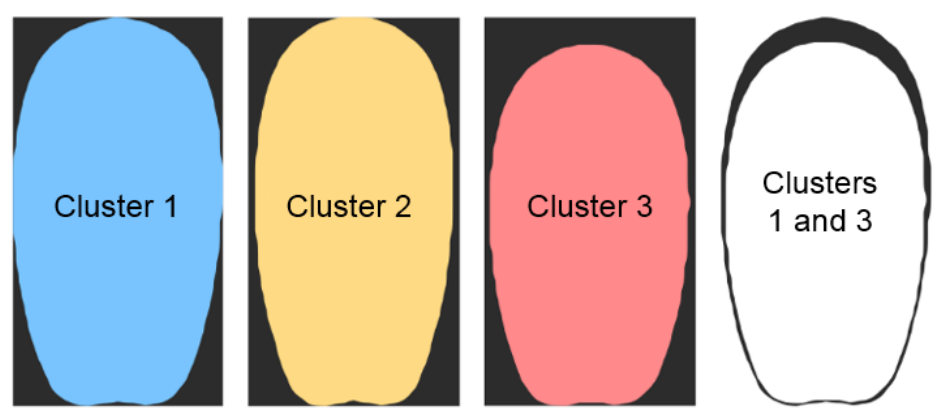

246 Figure 3: Analysis of the training group identifies distinct morphological clusters. (A) Cluster analysis 
that cluster that are from fertile animals and the difference in representation from that expected by

Comparison of these clusters indicates that the cluster in which sperm heads from fertile animals are

A

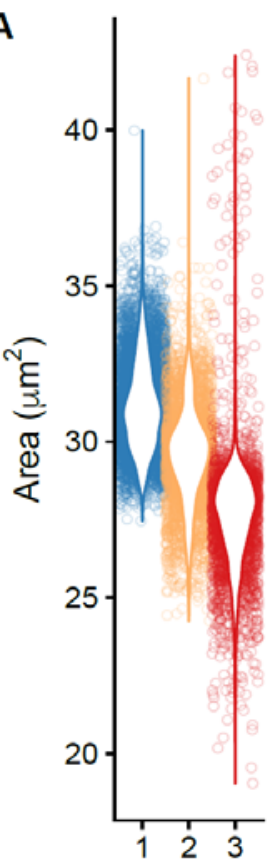

B

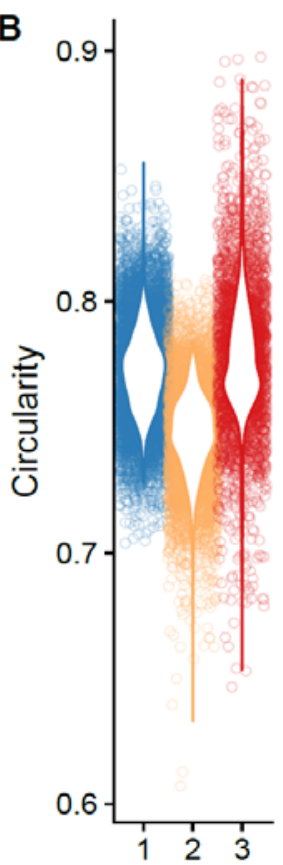

C

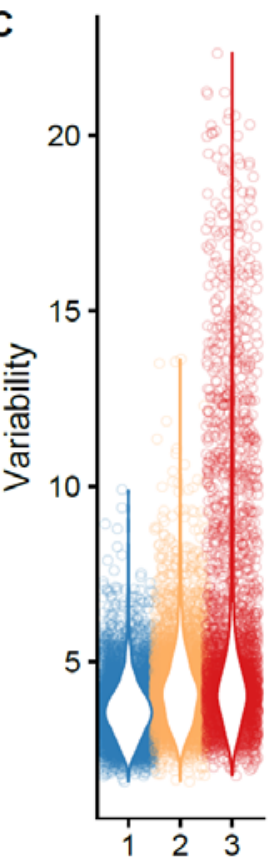

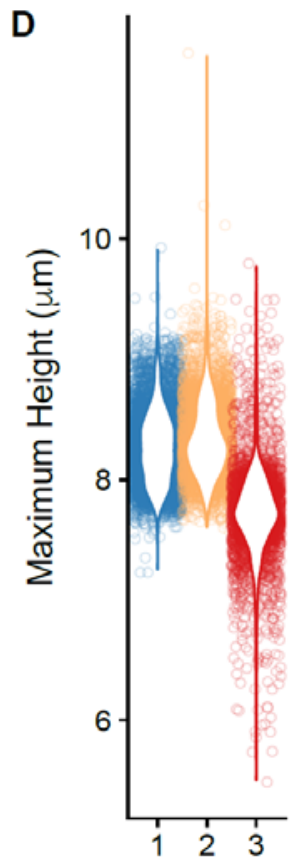

E

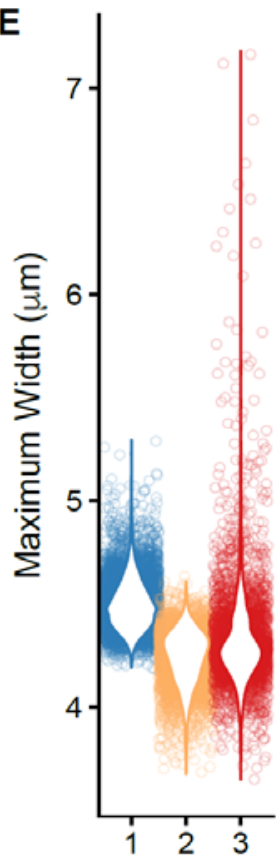

Figure 4: Sperm heads from the three clusters detected are morphologically distinct. Comparison of

(A) Area, with $1>2>3$ ( $p<0.001$ by pairwise post hoc test), (B) Circularity, with $3>1>2$, (C) Variability, 
with $3>2>1$, (D) Maximum Height, with $2>1>3$, and (E) Maximum width, with $1>3>2$, for the three clusters.

The three clusters identified in the training group were also recovered in the test group (Figure S5),

271 with analysis of sperm head morphology showing the same differences between clusters (data not

272 shown). As in the training group, sperm heads from fertile animals were overrepresented in cluster 1

273 and underrepresented in cluster 3 (Figure S5). This supports the idea that the frequency of certain

274 sperm head morphology types can be used to predict fertility. Several approaches were investigated

275 to test this. Firstly, we used the mean trait values for the three clusters identified in the training group

276 to define the initial partitions for a K-means clustering of the sperm heads from the test group of 20

277 fertile and 20 sub-fertile animals. The proportion of nuclei from each animal that was allocated to

278 each cluster was then determined and compared to the proportions determined from the training set

279 (Figure 5). This indicates that this method does accurately predict fertility in some animals, but that no scheme would correctly identify status for all animals. That is, if the aim was to exclude any animal where sub-fertility was suggested, then 8 of the 20 fertile animals and 6 of the 20 sub-fertile animals would be retained (Table S2). Giving better results, retaining all those animals where cluster membership suggested fertility would leave 13 of the 20 fertile animals and 6 of the 20 sub-fertile animals (Table S2). Similarly, attempts to predict fertility using other approaches - for example fertile animals or the exclusion of fertile animals. 
A

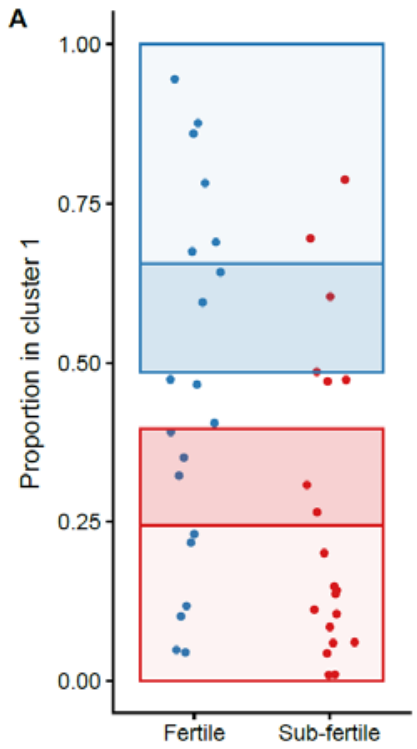

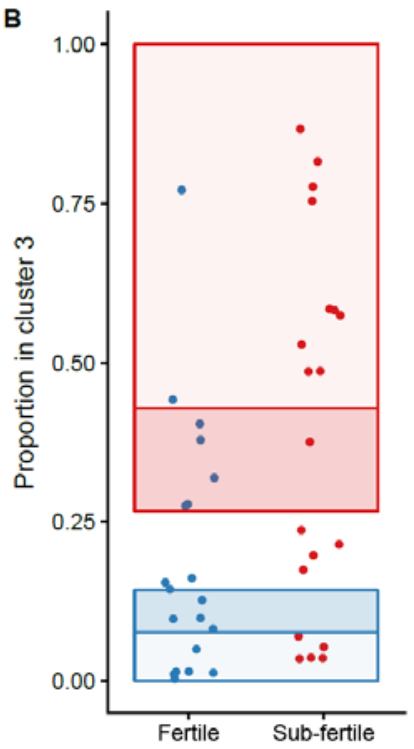

289 Figure 5: Cluster membership can predict fertility for some individuals. Shown are the proportion of sperm heads from each individual in the test group that are grouped into (A) cluster 1 and (B) cluster

3. Dark shaded rectangles highlight the $95 \%$ confidence intervals from cluster representation in the training set. Light shaded rectangles highlight areas between these confidence intervals and either 0 or 1. Red points falling into the region defined by the blue rectangles therefore, represent sub-fertile animals that would be classified as fertile and vice versa for blue points falling inside the regions defined by red rectangles.

\section{Discussion}

298 Over the last decade, Al has become commonplace in the pig breeding industry; it is therefore, an economical imperative to identify boars with prime fertility. To this end, various computer technologies have been developed, or adapted, to allow quantitative analysis of boar sperm characteristics. Prime examples are the CASA systems that have been developed and improved over almost four decades; these are now widely used in semen handling centres and spermatology laboratories. Currently, the Sperm Class Analyser (SCA) is considered the gold standard in automated sperm morphology analysis (ASMA) systems (Horst, 2015). SCA has been shown to provide accurate measurements of head, mid-piece and tail morphometry in several mammalian species including 
humans (Soler et al., 2003), horses (Hidalgo et al., 2005) and goats (Hidalgo et al., 2006). The absence of standardised morphometric parameters and corresponding values which could be used to identify both normal and abnormal sperm however, is a limitation which is common to most species (Horst, 2015).

311 We previously developed the Nuclear Morphology Analysis software for rapid and accurate assessment of nuclear morphology in mouse lines (Skinner et al., 2018). In contrast to other morphometric approaches, such as elliptic Fourier analysis (Ostermeier et al., 2001), or Procrustesbased geometrics (Varea Sánchez et al., 2013), our analysis can be run rapidly on many thousands of nuclei, using automatic detection of landmarks and semi-landmarks within the nucleus, and the results map cleanly back to the biological structure of the nucleus. In this study we have extended the capability of the software to recognise pig sperm. The ability to distinguish subtle morphological differences in pig sperm demonstrate the utility of this approach for other spatulate nuclei, as commonly found in other mammalian species, including humans (Skinner and Johnson, 2017).

Here we have used this software to analyse sperm head morphology in pigs assessed as either fertile

327 fertility boars had less elongated sperm heads that were significantly smaller than those of lower 328 fertility (non-return rate lower than 86\%) (Hirai et al., 2001). This study used the relationship between

329 fertility - based on non-return rate, which was expressed as a percentage of sows who were not chosen for a second insemination between 60 and 90 days following the first insemination - and the 
2001). This may represent a difference between breeds as it is known that sperm head dimensions differ between breeds; for example, the average sperm head area of Landrace and Large White sperm is 34.4 and $34.7 \mu \mathrm{m}^{2}$, respectively (Saravia et al., 2007).

Our analysis also identifies three clusters of morphology types (Figure 3). These clusters group sperm

337 heads that have a low variability and a high area (cluster 1 ), that are tall and narrow (cluster 2), and 338 those that have a low area and a high variability (cluster 3) (Figure 4). This mirrors the clusters identified by previous work on sperm head morphology in pigs that used measures of area, height (or length) and width (Hirai et al., 2001; Vicente-Fiel et al., 2013a, b). Here however, our analysis also indicates that the variability differs between these groups (Figure 4). We hypothesised that a certain type of sperm head might only be present, or be overrepresented, in semen from sub-fertile animals.

Whilst it is expected that such differences in sperm head morphology would have an impact on the cluster 3 , i.e. sub-fertile animals have a high incidence of sperm heads a low area and a high variability

(Figure 4).

350 Given that chromatin packaging in the sperm nucleus has been shown to be vital for a successful pregnancy, the morphological differences may be associated with poor chromatin packaging, due to potential DNA damage and failure of sperm decondensation, which could result in fertilisation failure (Bianchi et al., 1996; Sakkas et al., 1996). Sperm subpopulations have also been identified based on biochemical parameters (Calamera et al., 2003; Buffone et al., 2004) and it would be of interest to of morphology. 
358 Given that automated approaches to identify sub-fertile individuals would be of value in pig production, we sought to determine if our measures of morphology could be used to predict fertility. morphology differs between breeds. Secondly, the assessment of animals as either fertile or subfertile means that more quantitative differences between samples cannot be factored into the analysis. For example, a sample with a morphology score of $70 \%$ and a motility score of 4 would be classified as fertile whilst one with a morphology score of $80 \%$ and a motility score of 3 would be classified as sub-fertile.

In conclusion, here we have shown that high-throughput morphometric analysis of pig sperm reveals suggest several ways that this work can be extended to look at the variation within individuals over time and between both individuals and breeds. As some studies have also suggested that routinely assessed sperm parameters (morphology, motility and concentration) are not entirely indicative of

380 fertility or prolificacy (Gadea, 2005; O'Connor et al., 2017), is becoming apparent that more detailed 381 investigation of chromatin organisation is necessary. We aim to extend our analyses to determine whether chromatin compaction or chromosome position in pig sperm varies between fertile and subfertile samples. 
Declaration of interest

386 None of the authors of this paper has a financial or personal relationship with other people or

387 organisations that could inappropriately influence or bias the content of the paper.

388

$389 \quad$ Funding

390 BMS was supported by the Leverhulme Trust (grant RPG337) and the Biotechnology and Biological

Sciences Research Council (BBSRC, grant BB/N000129/1).

392

393 Acknowledgments

394 We thank Canterbury Christ Church University for supporting AM, SH and KF. We would also like to

395 thank JSR Genetics Ltd. for supplying samples used in this study.

396

397 Authors' contributions

398 Conceptualisation, BMS and KF; Methodology, BMS, KF, SH; Software and Validation, BMS;

399 Investigation, AM; Data Curation and Formal Analysis, AM, SH; Visualisation, SH, BMS; Supervision and

400 Project Administration, KF, SH; Writing - Original Draft, AM, KF; Writing - Review and Editing, BMS, KF,

401 AM, SH; Resources, KF, GW. All authors gave final approval for publication.

402

403 
404

405

406

407

408

409

410

\section{References}

Agarwal A, Deepinder F, Sharma RK, Ranga G and Li J (2008) Effect of cell phone usage on semen analysis in men attending infertility clinic: an observational study. Fertility and Sterility 89 124128.

Apostoli P, Kiss P, Porru S, Bonde JP and Vanhoorne M (1998) Male reproductive toxicity of lead in animals and humans. Occup Environ Med 55 364-374.

Auger J, Jouannet P and Eustache F (2016) Another look at human sperm morphology. Human Reproduction 31 10-23.

Bianchi PG, Manicardi GC, Urner F, Campana A and Sakkas D (1996) Chromatin packaging and morphology in ejaculated human spermatozoa: evidence of hidden anomalies in normal spermatozoa. Molecular Human Reproduction 2 139-144.

Buffone MG, Doncel GF, Briggiler CIM, Vazquez-Levin MH and Calamera JC (2004) Human sperm subpopulations: relationship between functional quality and protein tyrosine phosphorylation. Human Reproduction 19 139-146.

Calamera J, Buffone M, Ollero M, Alvarez J and Doncel GF (2003) Superoxide dismutase content and fatty acid composition in subsets of human spermatozoa from normozoospermic, asthenozoospermic, and polyzoospermic semen samples. Molecular Reproduction and Development 66 422-430.

Coetzee K, Kruge TF and Lombard CJ (1998) Predictive value of normal sperm morphology: A structured literature review. Human Reproduction Update 4 73-82.

Cooper TG, Noonan E, von Eckardstein S, Auger J, Baker HWG, Behre HM, Haugen TB, Kruger T, Wang C, Mbizvo MT et al. (2009) World Health Organization reference values for human semen characteristics. Human Reproduction Update 16 231-245.

Curry M (2000) Cryopreservation of semen from domestic livestock. Reviews of Reproduction 5 4652.

Dziuk PJ and Henshaw G (1958) Fertility of boar semen artificially inseminated following in vitro 
Eliasson R (1971) Standards for investigation of human semen. Andrologia 3 49-64.

432

433

Feitsma H (2009) Artificial insemination in pigs, research and developments in The Netherlands, a review. Acta Scientiae Veterinariae 37 61-72.

Gadea J (2005) Sperm factors related to in vitro and in vivo porcine fertility. Theriogenology 63 431444.

Gerrits RJ, Lunney JK, Johnson LA, Pursel VG, Kraeling RR, Rohrer GA and Dobrinsky JR (2005) Perspectives for artificial insemination and genomics to improve global swine populations. Theriogenology 63 283-299.

Guzick D, Overstreet J, Factor-Litvak P, Brazil C, Nakajima S, Coutifaris C, Carson S, Cisneros P, Stenkampf M, Hill J et al. (2001) Sperm morphology, motility, and concentration in fertile and infertile men. The New England Journal of Medicine 345 1388-1393.

Hartman CG, Schoenfeld C and Copeland E (1964) Individualism in the Semen Picture of Infertile Men. Fertility and Sterility 15 231-253.

Hidalgo M, Rodriguez I, Dorado J, Sanz J and Soler C (2005) Effect of sample size and staining methods on stallion sperm morphometry by the Sperm Class Analyzer. Veterinarni Medicina 50 24-32.

Hidalgo M, Rodríguez I and Dorado J (2006) Influence of staining and sampling procedures on goat sperm morphometry using the Sperm Class Analyzer. Theriogenology 66 996-1003.

Hirai M, Boersma A, Hoeflich A, Wolf E, Foll J, Aumuller R and Braun J (2001) Objectively measured sperm motility and sperm head morphometry in boars (Sus scrofa): Relation to fertility and seminal plasma growth factors. Journal of Andrology 22 104-110.

Holt W V. and Van Look KJW (2004) Concepts in sperm heterogeneity, sperm selection and sperm competition as biological foundations for laboratory test of semen quality. Reproduction $\mathbf{1 2 7}$ $527-535$.

Horst G Van Der (2015) Automated sperm morphology analysis. Proceedings of the $25^{\text {th }}$ Technical Conference on Artificial Insemination \& Reproduction 125-128. 
Huggins C, Scott WW and Heinen JH (1942) Chemical composition of human semen and of the secretions of the prostate and seminal vesicles. American Journal of Physiology - Legacy Content 136 467-473.

Johnson LA, Aalbers JG, Willems CMT and Sybesma W (1981) Use of boar spermatozoa for artificial insemination and fertilizing capacity of fresh and frozen spermatozoa in sows on 36 farms. Journal of Animal Science 52 1130-1136.

Jung A and Schuppe HC (2007) Influence of genital heat stress on semen quality in humans. Andrologia $39203-215$.

King GJ and Macpherson JW (1973) A Comparison of Two Methods for Boar Semen Collection. Journal of Animal Science 36 563-565.

López Rodríguez A, Rijsselaere T, Beek J, Vyt P, Van Soom A and Maes D (2013) Boar seminal plasma components and their relation with semen quality. Systems Biology in Reproductive Medicine 59 $5-12$.

MacLeod J and Gold RZ (1951) The male Factor in fertility and infertility. Fertility and Sterility 2 394414.

Maes D, Nauwynck H, Rijsselaere T, Mateusen B, Vyt P, de Kruif A and Van Soom A (2008) Diseases in swine transmitted by artificial insemination: An overview. Theriogenology 70 1337-1345.

Maree L, Du Plessis SS, Menkveld R and Van Der Horst G (2010) Morphometric dimensions of the human sperm head depend on the staining method used. Human Reproduction 25 1369-1382. sperm morphology in humans. Mutation Research Letters 207 159-164.

Menkveld R and Kruger TF (1996) Evaluation of sperm morphology by light microscopy. Human Spermatozoa in Assisted Reproduction 89-107. errors in the pig genome assembly. Animal Genetics 48 395-403. 
Ostermeier GC, Sargeant GA, Yandell BS, Evenson DP and Parrish JJ (2001) Relationship of bull fertility to sperm nuclear shape. Journal of Andrology 22 595-603.

Overstreet JW and Katz DF (1987) Semen analysis. The Urologic Clinics of North America 14 441-449.

Owen DH (2005) A Review of the physical and chemical properties of human semen and the formulation of a semen simulant. Journal of Andrology 26 459-469.

Paz GF, Sofer A, Homonnai ZT and Kraicer PF (1977) Human semen analysis: seminal plasma and prostatic fluid compositions and their interlations with sperm quality. International Journal of Fertility 22 140-147.

Perreault SD, Aitken RJ, Baker HWG, Evenson DP, Huszar G, Irvine DS, Morris ID, Morris RA, Robbins WA, Sakkas D et al. (2003) Integrating new tests of sperm genetic integrity into semen analysis:

Polge C, Day B and Groves T (1968) Synchronisation of ovulation and artificial insemination in pigs. Veterinary Record 83 136-142.

R Core Team (2018) R: a language and environment for statistical computing. R Foundation for Statistical Computing, Vienna, Austria. URL, https://www.R-project.org/

Roca J, Vázquez JM, Gil MA, Cuello C, Parrilla I and Martínez EA (2006) Challenges in pig artificial insemination. Reproduction in Domestic Animals 41 43-53.

Rowe P, Comhaire F, Hargreave T and Mellows H (1993) WHO manual for the standardized investigation and diagnosis of the infertile couple. Cambridge, England: Cambridge University Press.

Sakkas D, Urner F, Bianchi PG, Bizzaro D, Wagner I, Jaquenoud N, Manicardi G and Campana A (1996) morphometry are not related to chromatin integrity. Theriogenology 68 196-203. 
508

509

510

511

512

513

514

515

516

517

518

519

520

521

522

523

524

525

526

527

528

529

530

531

532

533

Skinner BM and Johnson EE (2017) Nuclear morphologies: their diversity and functional relevance. Chromosoma 126 195-212.

Skinner BM, Rathje CC, Bacon J, Johnson EEP, Larson EL, Kopania EEK, Good JM, Yousafzai G, Affara NA and Ellis PJI (2018) A high-throughput method for unbiased quantitation and categorisation of nuclear morphology. bioRxiv 312470, doi: 10.1101/312470.

Singleton WL (2001) State of the art in artificial insemination of pigs in the United States. Theriogenology 56 1305-1310.

Soler C, De Monserrat JJ, Gutiérrez R, Nuñez J, Nuñez M, Sancho M, Pérez-Sánchez F and Cooper TG (2003) Use of the sperm-class analyser ${ }^{\circledR}$ for objective assessment of human sperm morphology. International Journal of Andrology 26 262-270.

Tardif S, Laforest JP, Cormier N and Bailey JL (1999) The importance of porcine sperm parameters on fertility in vivo. Theriogenology 52 447-459.

U.S. Environmental Protection Agency . (1996) Guidelines for reproductive toxicity risk assessment. Environmental Protection Agency 61 56274-56322.

Varea Sánchez M, Bastir M and Roldan ERS (2013) Geometric morphometrics of rodent sperm head shape. PLOS ONE 8 1-10.

Vicente-Fiel S, Palacín I, Santolaria P, Hidalgo CO, Silvestre MA, Arrebola F and Yániz JL (2013a) A comparative study of the sperm nuclear morphometry in cattle, goat, sheep, and pigs using a new computer-assisted method (CASMA-F). Theriogenology 79 436-442.

Vicente-Fiel S, Palacín I, Santolaria P and Yániz JL (2013b) A comparative study of sperm morphometric subpopulations in cattle, goat, sheep and pigs using a computer-assisted fluorescence method (CASMA-F). Animal Reproduction Science 139 182-189.

WHO (2010) Examination and processing of human semen. URL, http://whqlibdoc.who.int/publications/2010/9789241547789_eng.pdf 
Figure legends

535 Figure 1: A representative summary of analysis workflow. (A) DAPI stained nucleus from a fertile boar at $1000 x$ magnification. The software measures the interior angles along the perimeter of the nucleus as represented by point 1-6. (B) Schematic showing how these angles generate a profile. The figure shows the median and interquartile rages for one fertile sperm sample, and has been segmented at

540 local maxima and minima. (C) The consensus fertile pig sperm head shape, showing positions of profile segments in the nucleus.

Figure 2: Comparison of sperm head area between 50 fertile (blue) and 50 sub-fertile (red) boars. (A)

544 Area of 11,534 sperm heads from fertile animals (blue) and 11,326 sperm heads from sub-fertile animals (red). (B) Individual Tufte boxplots of sperm head area for 50 fertile (blue) and 50 sub-fertile (red) boars.

548 Figure 3: Analysis of the training group identifies distinct morphological clusters. (A) Cluster analysis identifies three distinct clusters. Shown below each cluster is the percentage of sperm heads within that cluster that are from fertile animals and the difference in representation from that expected by chance (i.e. a positive value indicates that fertile nuclei are overrepresented in a cluster and vice versa

554 for a negative value). (B) The consensus shapes of the three clusters demonstrate the separation of 555 nuclei on size and shape. Cluster 1 contains the majority of the fertile sperm; cluster 2 has equal 556 representation of fertile and sub-fertile sperm; cluster 3 predominantly contains sub-fertile sperm.

557 The increasing compaction of the nuclei is readily apparent overlaying consensus nuclei from clusters 1 and 3. 
560 Figure 4: Sperm heads from the three clusters detected are morphologically distinct. Comparison of

561 (A) Area, with $1>2>3$ ( $p<0.001$ by pairwise post hoc test), (B) Circularity, with $3>1>2$, (C) Variability,

562 with $3>2>1$, (D) Maximum Height, with $2>1>3$, and (E) Maximum width, with $1>3>2$, for the three

563 clusters.

564

565 Figure 5: Cluster membership can predict fertility for some individuals. Shown are the proportion of

566 sperm heads from each individual in the test group that are grouped into (A) cluster 1 and (B) cluster

567 3. Dark shaded rectangles highlight the $95 \%$ confidence intervals from cluster representation in the

568 training set. Light shaded rectangles highlight areas between these confidence intervals and either 0

569 or 1. Red points falling into the region defined by the blue rectangles therefore, represent sub-fertile

570 animals that would be classified as fertile and vice versa for blue points falling inside the regions

571 defined by red rectangles.

572 
bioRxiv preprint doi: https://doi.org/10.1101/314708; this version posted May 4, 2018. The copyright holder for this preprint (which was not certified by peer review) is the author/funder, who has granted bioRxiv a license to display the preprint in perpetuity. It is made available under aCC-BY 4.0 International license.

\section{$573 \quad$ Figures}

A

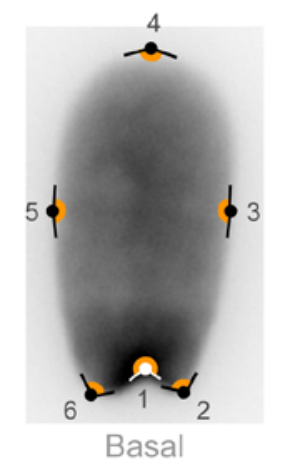

574

B

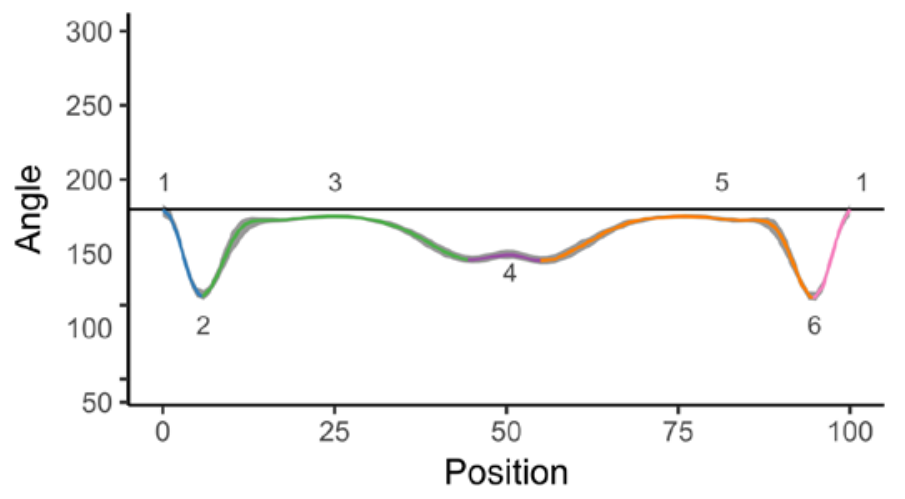

C

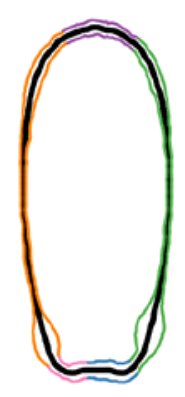

575

Figure 1

576 
bioRxiv preprint doi: https://doi.org/10.1101/314708; this version posted May 4, 2018. The copyright holder for this preprint (which was not certified by peer review) is the author/funder, who has granted bioRxiv a license to display the preprint in perpetuity. It is made available under aCC-BY 4.0 International license.

A

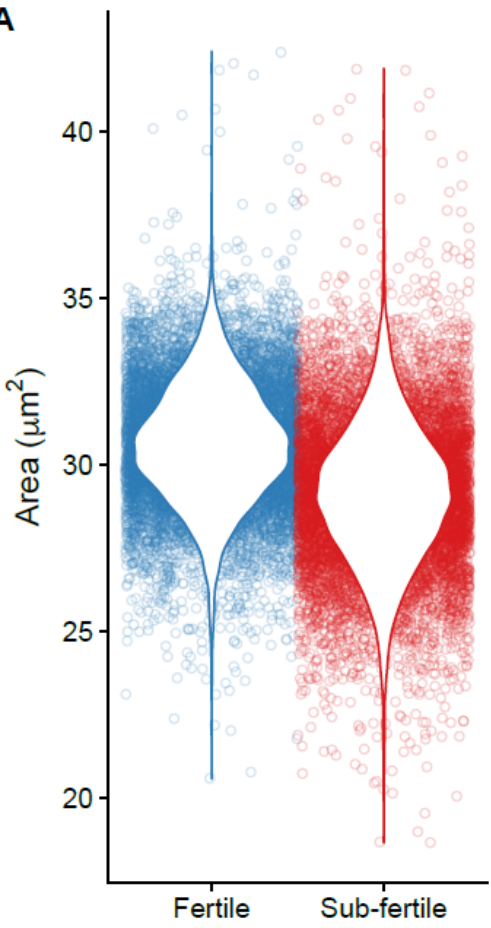

B

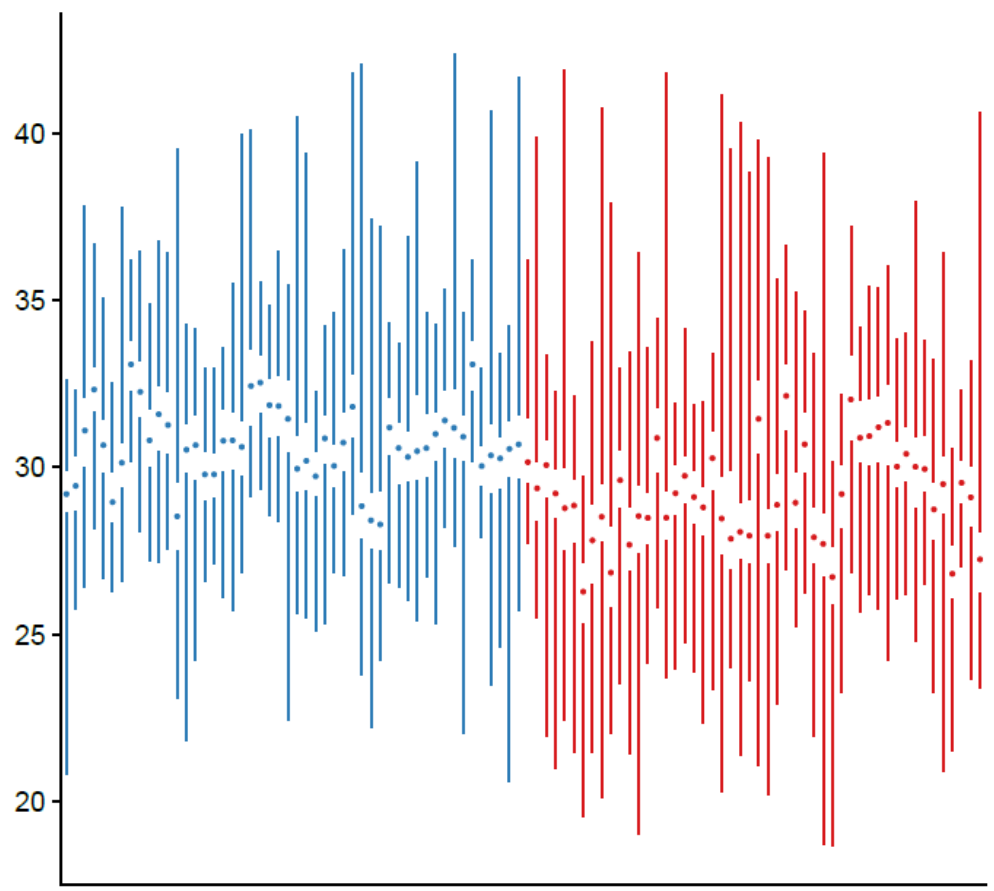

\section{$578 \quad$ Figure 2}


bioRxiv preprint doi: https://doi.org/10.1101/314708; this version posted May 4, 2018. The copyright holder for this preprint (which was not certified by peer review) is the author/funder, who has granted bioRxiv a license to display the preprint in perpetuity. It is made available under aCC-BY 4.0 International license.

A

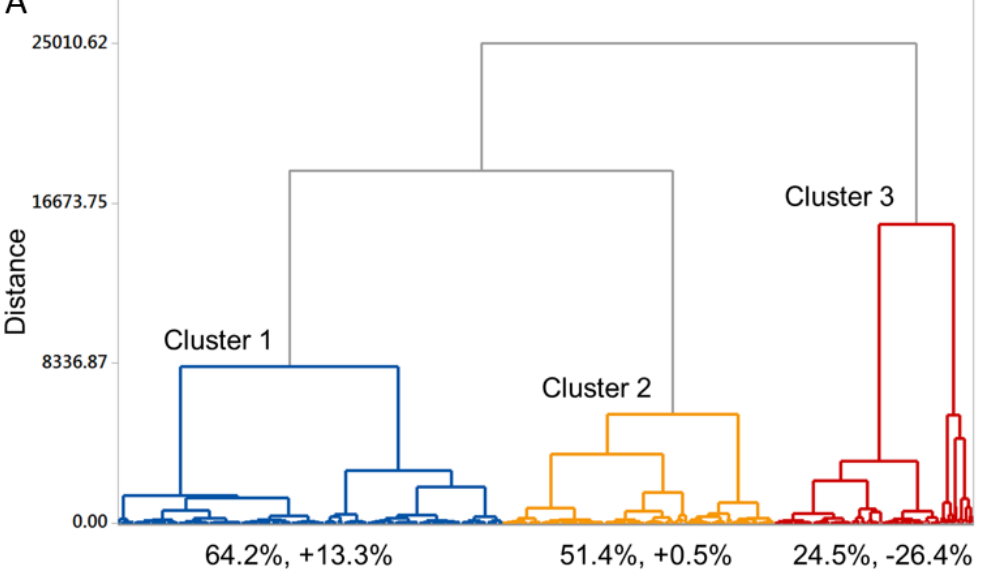

B

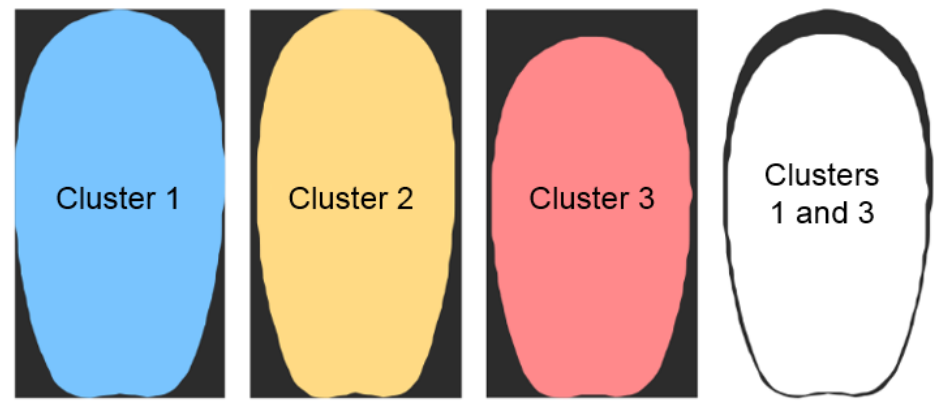

$581 \quad$ Figure 3

582 
A

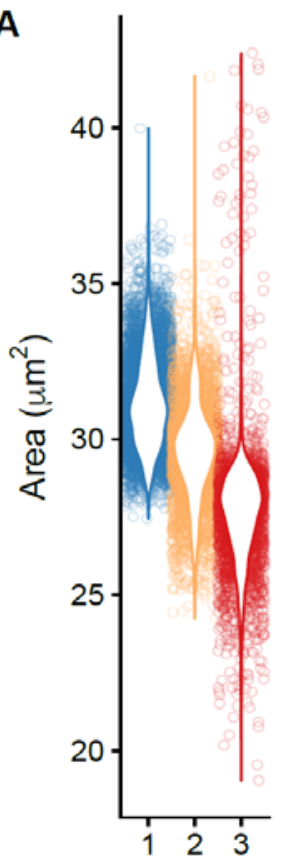

B

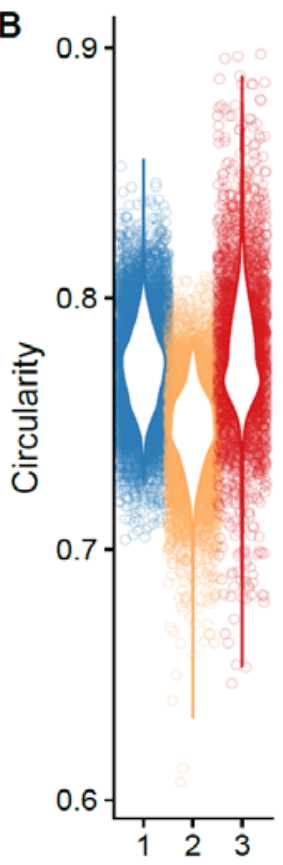

C

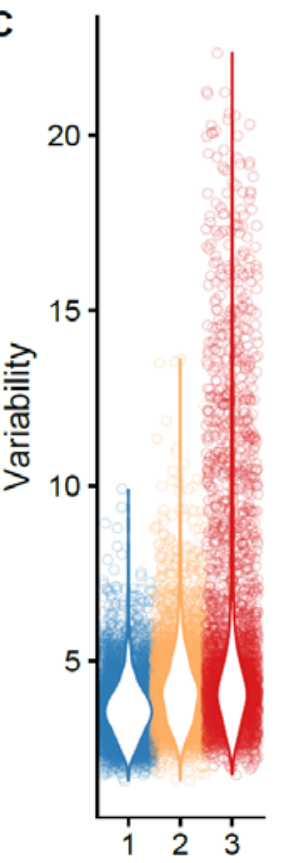

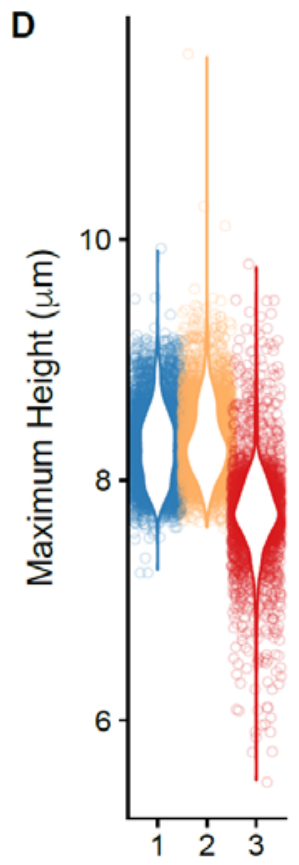

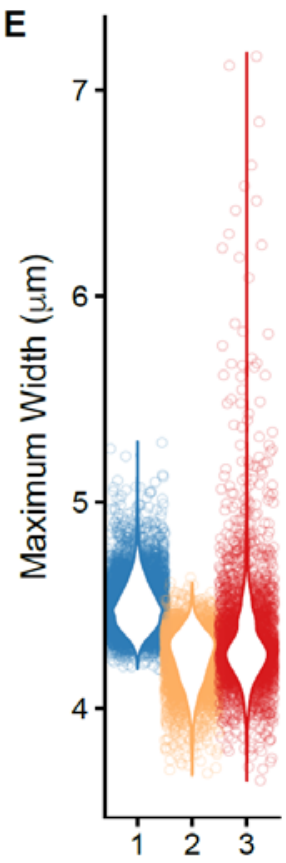

E

$584 \quad$ Figure 4

585 
bioRxiv preprint doi: https://doi.org/10.1101/314708; this version posted May 4, 2018. The copyright holder for this preprint (which was not certified by peer review) is the author/funder, who has granted bioRxiv a license to display the preprint in perpetuity. It is made available under aCC-BY 4.0 International license.

586
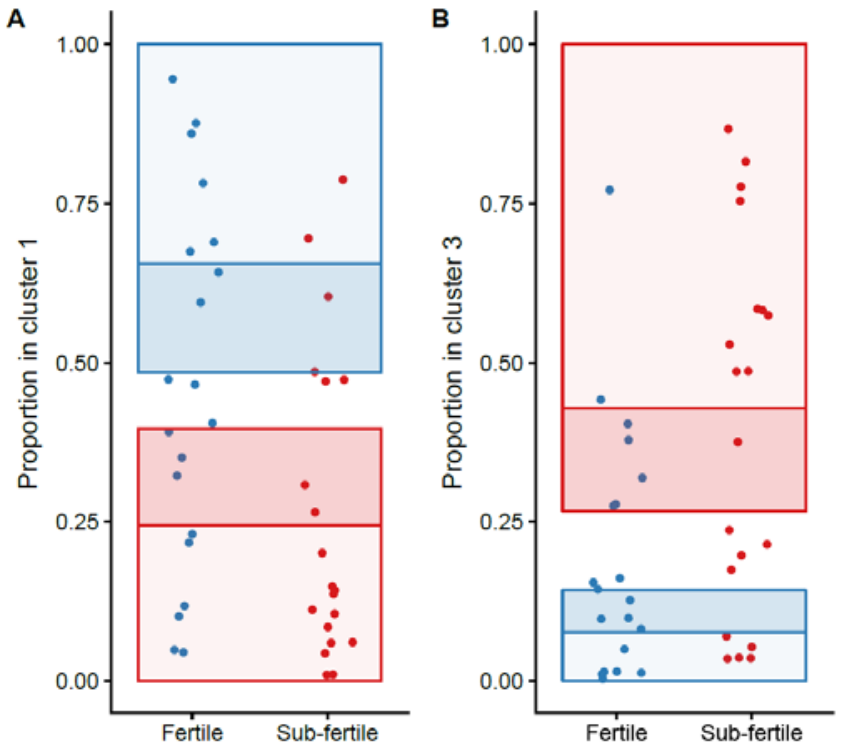

\section{$587 \quad$ Figure 5}

